\title{
Medikamente in der Schwangerschaft Welche Substanzen eignen sich im Akutfall?
}

Dirk Nauheimer

\begin{abstract}
Viele Medikamente sind plazentagängig - und stellen somit ein Risiko für das ungeborene Kind dar. Trotzdem lässt es sich nicht immer vermeiden, einer Schwangeren Medikamente zu verordnen: z. B. bei ungeplanten Krankenhausaufenthalten oder in der Geburtshilfe. Dabei muss man jedoch die physiologischen Veränderungen während der Schwangerschaft sowie die möglichen negativen Effekte häufig eingesetzter Substanzen auf Mutter und Fetus berücksichtigen. Nur so kann man die Anwendung so sicher wie möglich gestalten.
\end{abstract}

\section{Grundlagen}

Medikamente zurückhaltend verordnen Die Verabreichung von Medikamenten in der Schwangerschaft oder zur Geburtshilfe sollte aus Verantwortung gegenüber Mutter und Kind sehr bedacht erfolgen. Die Plazenta stellt eine nur eingeschränkte Barriere für pharmakologische Substanzen zwischen mütterlichem und fetalem Kreislauf dar. Da sich nahezu alle eingenommenen Medikamente der Mutter auch auf das Ungeborene auswirken, sollten potenziell fruchtgefährdende Arzneimittel ( $\bullet$ Tab. 1 ) vermieden werden und eine generelle Einnahme nur zurückhaltend erfolgen. Trotzdem kommt man nicht immer darum herum, Medikamente zu verordnen.

Gründe für Medikamentengabe in der Schwangerschaft Häufig notwendig wird dies im Rahmen von ungeplanten Krankenhausaufenthalten während der Schwangerschaft oder zu geburtshilflichen Maßnahmen.

- Etwa 0,5-2\% aller Schwangeren müssen sich pro Jahr einem nicht gynäkologischen Eingriff unterziehen [2].

- Zudem steigt die Rate der Schnittentbindungen an: 2008 erfolgten in Deutschland nahezu 30\% der 663000 Entbindungen per Kaiserschnitt. Dies entspricht einer Steigerung um etwa 12\% in den letzten 10 Jahren [3]. In speziellen neonatologischen Zentren liegt die Rate der Schnittentbindungen bereits bei bis zu $40 \%$ [4]. Eine Indikationsstellung zur Verabreichung von Medikamenten während der Schwangerschaft erfolgt allerdings nicht nur aufgrund operativer Eingriffe, sondern hat vielfältigste Ursachen.

- Häufig bestehen bereits Verordnungen häuslicher Medikation.

- Gelegentlich entwickeln sich schwangerschaftsspezifische Komplikationen, die eine Einnahme von Präparaten erfordern.

Was sollte man beachten? Aufgrund mangelnder Routine mit diesem speziellen Patientenkollektiv treten häufig Unsicherheiten bezüglich physiologischer Veränderungen, sowie Wirkungen und Wechselwirkungen der Medikamente auf. Oft herrscht Unklarheit über die Anwendbarkeit verschiedener Pharmaka im Rahmen einer Schwangerschaft. Zu bedenken sind bei der Auswahl der Medikamente, der anästhesiologischen Prozedur und Adjuvanzien:

- Einflüsse auf die uteroplazentare Perfusion

- teratogene oder mutagene Effekte

- Induktion von Abort oder Frühgeburtlichkeit

- postpartale Adaptationsstörungen (kardiozirkulatorische oder neuromuskuläre Depression)

Im Falle der Anwendung von Pharmaka während einer Schwangerschaft gilt es nicht nur, die Erkrankung der Mutter sicher und erfolgreich zu behandeln, sondern auch, unerwünschte nachteilige Effekte für das ungeborene Kind zu vermeiden.

\section{Besonderheiten bei Eingriffen}

$\nabla$

Anästhesie bei Sectio Eine nationale deutsche Umfrage aus dem Jahr 2005 zeigte, dass

- zur elektiven Schnittentbindung neuroaxiale Verfahren der Allgemeinanästhesie vorgezogen werden [5].

Notfallsituationen, mütterliche Koagulopathie, regionale Kontraindikationen, Sepsis, das Versagen regionaler Verfahren oder Ablehnung durch die Patientin stellen aber weiterhin Indikationen zur Durchführung einer Allgemeinanästhesie dar. 
- Bei dringlicher Indikation oder im Rahmen von Notfallsectiones wird zu 86-99\% eine Intubationsnarkose bevorzugt [6, 7]. Hierzu stellt die endotracheale Intubation nach einer „rapid sequence induction“ (RSI) das Verfahren der Wahl zur Einleitung und Sicherung des Atemwegs dar [5].

Vorteile bieten die möglichst rasche Sicherung des Atemwegs und die geringere Rate an Kreislaufinstabilitäten im Vergleich zu regionalen Verfahren. Nachteile ergeben sich meist aus den Komplikationen während der Sicherung des Atemwegs.

Cave Bei Intubationsnarkosen stehen Komplikationen wie Intubationsschwierigkeiten mit Hypoxämie, Aspiration und Awareness im Vordergrund.

OP birgt Gefahr für Fehlgeburten Unter den nicht gynäkologischen Eingriffen bei Schwangeren ist das akute Abdomen mit einer Inzidenz von $0,1-0,4 \%$ beschrieben. Hier überwiegt die akute Appendizitis mit einer Inzidenz von 0,05-0,1\%. Eine symptomatische Cholezystolithiasis $(<0,1 \%)$ ist bei ca. 2-4\% Gallensteinträgern mit einer sich anschließender Cholezystektomie seltener.

- Je nach Erkrankungsschwere sind Abortraten von $0-18 \%$ beschrieben [8].

Auch ein akutes Abdomen aufgrund eines Ileus ist ein seltenes Krankheitsbild bei Schwangeren (0,01-0,03\%). Als Ursache findet sich zumeist eine mechanische Störung der Darmpassage bei Briden oder Verwachsungen.

- Zu beachten ist, dass bei Eingriffen im 1. Trimenon die Abortrate am höchsten ist und

- dass bei Eingriffen im 3. Trimenon mit einer erhöhten Rate an Frühgeburtlichkeit (teilweise bis $8 \%$ ) gerechnet werden muss [9].

Die mütterliche Mortalität ist bei chirurgischen Eingriffen insgesamt eher gering und wird mit $<1: 10000$ angegeben.

\section{Physiologische Veränderungen während der Schwangerschaft}

Kardiovaskuläres System Das Herzzeitvolumen (HZV) nimmt während der Schwangerschaft um ca. 30-40\% zu, wobei weniger die Herzfrequenz ansteigt als das Schlagvolumen (+30\%). Der systemische vaskuläre Widerstand (SVR) nimmt aufgrund reduzierter Vasopressin- und Angiotensinsensitivität ab [10].

Auch das Blutvolumen steigt um etwa 30-40\%. Hier erhöht sich v.a. das Plasmavolumen, während sich korpuskuläre Anteile nicht in gleichem Maße verändern.

- Es resultiert eine Hämodilution mit scheinbarer Schwangerschaftsanämie.

Plasmaproteine Auch die Plasmaproteine erfahren eine gesteigerte Synthese, jedoch in Rela-

tion zur Zunahme des Plasmavolumens quantitativ geringer.

- Durch diese relative Hypoproteinämie verringert sich der kolloidosmotische Druck (KOD) und bedingt eine generalisierte Ödemneigung.

- Als Folge der Schwellung von Schleimhäuten im pharyngo-laryngealen Bereich ist mit einer erhöhten Inzidenz an erschwerten Intubationen (etwa $4 \%$ ) zu rechnen [11].

Gerinnung Eine Hyperkoagulabilität ist die Folge einer vermehrten Synthese der Gerinnungsfaktoren I, II, VII, VIII, IX, X und XII. Dies soll gegen Blutverluste schützen, ist aber auch häufig die Ursache für thrombembolische Ereignisse. Begünstigt wird dies auch durch venöse Abflussbehinderung aus der unteren Körperhälfte durch den vergrößerten Uterus und einer möglichen cavalen Kompression.

Eine während der Schwangerschaft abnehmende Fibrinolyse verändert sich unter der Geburt in eine hyperfibrinolytische Aktivität [12].

Respiratorisches System Die Atmung ist gekennzeichnet durch eine Zunahme des Atemminutenvolumens (AMV) um ca. 40-50\% und eine gesteigerte alveoläre Ventilation um bis zu $65 \%$. Die Atemfrequenz steigt nur geringfügig an (bis zu 10\%). Grundlage hierfür ist der um etwa $20 \%$ gestiegene Sauerstoffverbrauch $\left(\mathrm{VO}_{2}\right)$. Durch die Größenzunahme des Uterus erhöht sich der intraabdominelle Druck und reduziert gleichzeitig die funktionelle Residualkapazität (FRC) um $20 \%$.

Tab. 1 Mod. nach [1].

\section{Embryotoxische Medikamente}

ACE-Hemmer

AT-II-Rezeptorantagonisten

Aminoglykoside

Benzodiazepine

(Langzeittherapie oder sub partu)

Chinolone

Chloramphenicol

Cumarine

Etilefrin

Etomidat

Immunsuppressiva

Opioide, Opiate

Na-Nitroprussid

Prilocain

Tetrazykline

Zytostatika
Anurie, Oligohydramnion, Kontrakturen, Schädelhypoplasie

Anurie, Oligohydramnion, Kontrakturen, Schädelhypoplasie

Oto-, Nephrotoxizität

Ateminsuffizienz, Floppy-Infant-Syndrom

Knorpelschäden möglich

Grey-Baby-Syndrom

Cumarinembryopathie

teratogene Effekte angenommen

(1. Trimenon)

Hemmung der Kortisolsynthese

Knochenmarkdepression

Entzugssyndrome, Ateminsuffizienz

Zyanidbelastung

Methämoglobinämie

Gelbfärbung der Zähne

Knochenmarkdepression 
Tab. 2 Mod. nach [13]. HZV: Herzzeitvolumen, AMV: Atemminutenvolumen, SVR: systemisch vaskulärer Widerstand,

FRC: funktionelle Residualkapazität,

GFR: glomeruläre Filtrationsrate,

$\mathrm{VO}_{2}$ : Sauerstoffverbrauch, $\mathrm{PaCO}_{2}$ : arterieller $\mathrm{CO}_{2}$-Partialdruck,

Hb: Hämoglobin,

KOD: kolloidosmotischer Druck, MAC: minimale alveoläre

Konzentration,

PChe: Plasmacholinesterase
- Insgesamt folgt daraus eine deutlich reduzierte Hypoxietoleranz [12].

Gastrointestinaltrakt Aufgrund des steigenden intraabdominellen Drucks, der Hyperazidität des Magensaftes, einer reduzierten Motilität des Magens sowie verringertem gastroösophagealem Sphinktertonus besteht eine erhöhte Gefahr zur Regurgitation und Aspiration.

Niere und glomeruläre Filtrationsrate Der renale Blutfluss sowie die glomeruläre Filtrationsrate (GFR) erhöhen sich während der Schwangerschaft um bis zu 50\%. Die GFR normalisiert sich zum 3. Trimenon wieder [13]. Es bestehen veränderte Tubulusbarrieren mit erhöhten Verlusten an Glukose und Aminosäuren.

Endokrinum Komplexe Änderungen in Hormonhaushalt und Stoffwechselprozessen bedingen Veränderungen des Kohlenhydrat-, Fett- und Proteinstoffwechsels zugunsten des fetalen Wachstums. Das führt zu einer diabetogenen Stoffwechsellage bei steigenden Insulinspiegeln und zunehmender relativer Insulinresistenz.

Uteroplazentare Perfusion Die uteroplazentare Perfusion nimmt im Verlauf der Schwangerschaft aufgrund des Wachstums der Plazenta zu. Gegen Ende des 3. Trimenons erreicht sie einen Blutfluss von etwa $500-700 \mathrm{ml} / \mathrm{min}$. Er dient dem Stoffaustausch zwischen Mutter und Feten.

- Es besteht keine autonome Regulation des Blutflusses, weshalb letztlich die uteroplazentare Perfusion vom mütterlichen Blutdruck abhängt.

Die uterinen Spiralarterien speisen den intervillösen Raum der Plazenta. Fetale Trophoblasten bilden an dieser Stelle zum intervillösen Raum die Plazentabarriere aus.

- Eine Änderung des HZV sowie lokale Veränderungen der Perfusion und Umverteilungsprozesse beeinflussen die Perfusion.

Vasokonstringierende endo- oder exogene Faktoren können durch den Einfluss des Endothels (erhöhte Produktion an NO oder Prostazyklin) vermindert werden $[10,12]$.

- Tab. 2 fasst die typischen Veränderungen in der Schwangerschaft noch einmal zusammen.

\section{Allgemeine Probleme und Schwierigkeiten \\ $\nabla$}

Off-label-Use Der Einsatz vieler Medikamente im Rahmen einer Schwangerschaft erfolgt ohne entsprechende Zulassung durch den Hersteller im sog. „Off-label-Use“. So finden sich regelmäßig Hinweise, dass die Applikation einer Substanz im Rahmen einer Schwangerschaft kontraindiziert ist, bzw. die Fachinformation führt eine Schwangerschaft unter Gegenanzeigen auf.

- Das liegt daran, dass für dieses Patientenkollektiv keine Zulassung erfolgen kann - denn Arzneimittel werden in aller Regel nicht an Schwangeren getestet. Gravidität stellt in allen Zulassungsstudien ein Ausschlusskriterium dar.

Der zulassungsüberschreitende Einsatz muss allerdings nicht rechtswidrig sein, wenn das zugelassene Medikament nach aktuellem Wissensstand hinreichend wirksam, die Indikation gegeben ist und eine gleichwertige Alternative nicht zur Verfügung steht [13].

Plazentapassage Die plazentare Austauschfläche beträgt zum Zeitpunkt der Geburt etwa $11 \mathrm{~m}^{2}$. Der Stoffaustausch erfolgt per diffusionem entlang eines Konzentrationsgradienten. In geringerem Maße sind auch aktive, Carrier-vermittelte Transportmechanismen beteiligt [2]. Physikochemische Eigenschaften, die einen Übertritt vom maternalen Blut in den fetalen Kreislauf begünstigen, sind:

- hoher Konzentrationsgradient

- hohe Lipophilie

- geringer Ionisierungsgrad

- geringe Proteinbindung

- geringes Molekulargewicht

Zudem beeinflussen anatomische sowie hämodynamische Eigenschaften der uteroplazentaren Einheit die Plazentapassage von Medikamenten. Hierzu zählen die Plazentareife, die plazentare Perfusion sowie der maternale und fetale SäureBasen-Haushalt.

Für viele Pharmaka stellt die Plazenta keine Barriere dar.

Auf einen Blick: physiologische Veränderungen während der Schwangerschaft

kardiovaskuläres System
- HZV $+30-40 \%$
- Schlagvolumen $+30 \%$
- Herzfrequenz $+20 \%$
- systolischer Blutdruck $-5 \%$
- diastolischer Blutdruck $-15 \%$
- SVR $-15 \%$
GFR $+50 \%$

kardiovaskuläres System respiratorisches System

- AMV +50\%

- Atemfrequenz $+10 \%$

- Atemzugvolumen $+40 \%$

- FRC $-20 \%$

- $\mathrm{VO}_{2}+20-30 \%$

- alveoläre Ventilation $+65 \%$

- $\mathrm{PaCO}_{2}-15 \%$

\section{hämatologische Parameter}

- Blutvolumen $+35 \%$

- Plasmavolumen $+45 \%$

- $\mathrm{Hb}-20 \%$

- Thrombozyten $-10 \%$

- Gerinnungsfaktoren $+30-50 \%$

- KOD reduziert

\section{Sonstiges}

- MAC reduziert

- PChe $-30 \%$ 


\section{Antibiotika und Antihistaminika}

\section{Antibiotika}

\section{$\nabla$}

Alle außer $\boldsymbol{\beta}$-Lactam-Antibiotika ungeeignet Penicilline und Cephalosporine können aufgrund fehlender teratogener Effekte eingesetzt werden. Kontraindiziert sind hingegen:

- Aminoglykoside, weil sie eine fetale Oto- und Nephrotoxizität aufweisen

- Tetrazykline, weil sie sich ab dem 2. Trimenon an Zähnen und Knochen anlagern - mit negativen Folgen für Knochenwachstum und Gelbfärbung der Zähne

- Chloramphenicol, weil es zum Grey-BabySyndrom führt

Auch von der Einnahme von Fluorchinolonen während der Schwangerschaft wird aufgrund möglicher Knorpelschäden abgeraten. Zwar gibt die aktuelle Datenlage keine Hinweise auf eine fetale oder neonatale Toxizität - in Tierversuchen wurde jedoch ein gestörtes Knorpelwachstum bei Ungeborenen und Jungtieren nachgewiesen [14].

\section{Antihistaminika}

$\boldsymbol{\nabla}$

Relativ sicher Die $\mathrm{H}_{2}$-Antagonisten Cimetidin und Ranitidin passieren bei niedrigem Molekulargewicht leicht die Plazenta, weisen aber weder teratogene noch mutagene Effekte auf. Sie können zur Reduktion der Magensaftazidität eingesetzt werden. Dimetinden als Vertreter der $\mathrm{H}_{1}$-Antagonisten kann während der gesamten Schwangerschaft Anwendung finden. Teratogene Effekte sind nicht bekannt. Vor der Einleitung einer Narkose neutralisiert man den Magensaft bei Patientinnen ab der 20. Schwangerschaftswoche häufig mit $30 \mathrm{ml}$ Natriumzitrat $(0,3 \mathrm{M})$. Auch dies gilt als unbedenklich [15].

\section{Schmerzmittel}

\section{Opiate \\ $\nabla$}

Gefahr der Kumulation beim Kind In Abhängigkeit von Lipophilie und Proteinbindung passieren Opiate die Plazenta unterschiedlich schnell und führen dosisabhängig zu postpartaler Ateminsuffizienz des Neugeborenen.

- Bei der Verwendung von Remifentanil fällt eine neonatale Depression vergleichsweise geringer aus. Grund ist die rasche Metabolisierung durch mütterliche, aber auch fetale Esterasen.

- Lipophile Opiate wie Fentanyl und Sufentanil neigen aufgrund ihrer kontextsensitiven Halbwertszeit bei repetitiver Anwendung zur Kumulation. Dadurch erhöht sich das Risiko einer Atemdepression des Neugeborenen.
Cave Die Halbwertszeiten von Opiaten können beim Fetus und Neugeborenen 5-7-mal länger ausfallen als beim Erwachsenen.

In der Epiduralanalgesie Auch epidurale Applikationen ( $\bullet$ Abb. 1 ) von Opiaten führen zu nachweisbaren Spiegeln im Fetus.

- Nachteilige Effekte für das Neugeborene sind für Sufentanil bei epiduraler Anwendung bis zu einer Tagesdosis von $30 \mu \mathrm{g}$ nicht zu erwarten. Dosisabhängig können „späte“ Atemdepressionen nach $12-24 \mathrm{~h}$ auftreten (bei Sufentanil seltener, etwas häufiger bei Morphin).

- Pethidin, das häufig in der Geburtshilfe eingesetzt wird, überwindet als lipophile und nur gering proteingebundene Substanz leicht die Plazentabarriere. Repetitiv angewandt, neigt es durch eingeschränkten Metabolismus des Neugeborenen zur Kumulation und zu Neugeborenendepression.

- Für Morphin wurden tierexperimentell teratogene Effekte mit ZNS-Fehlbildungen beschrieben, wohingegen beim Menschen keine teratogenen oder mutagenen Folgen bekannt wurden.

Mütterliche Hypotonien bei der Anwendung rückenmarksnaher Verfahren sind unbedingt zu überwachen und entsprechend zu therapieren [16].

Empfehlung für die Praxis Eine therapeutische, kurzfristige Opiatgabe während der Schwangerschaft wird bei strenger Indikationsstellung als unproblematisch angesehen. Geburtshilflich ist wegen der raschen Plazentapassage und der drohenden neonatalen Depression allerdings Vorsicht geboten.

- Besser ist es, mit der Applikation bis zur Abnabelung des Neugeborenen zu warten.

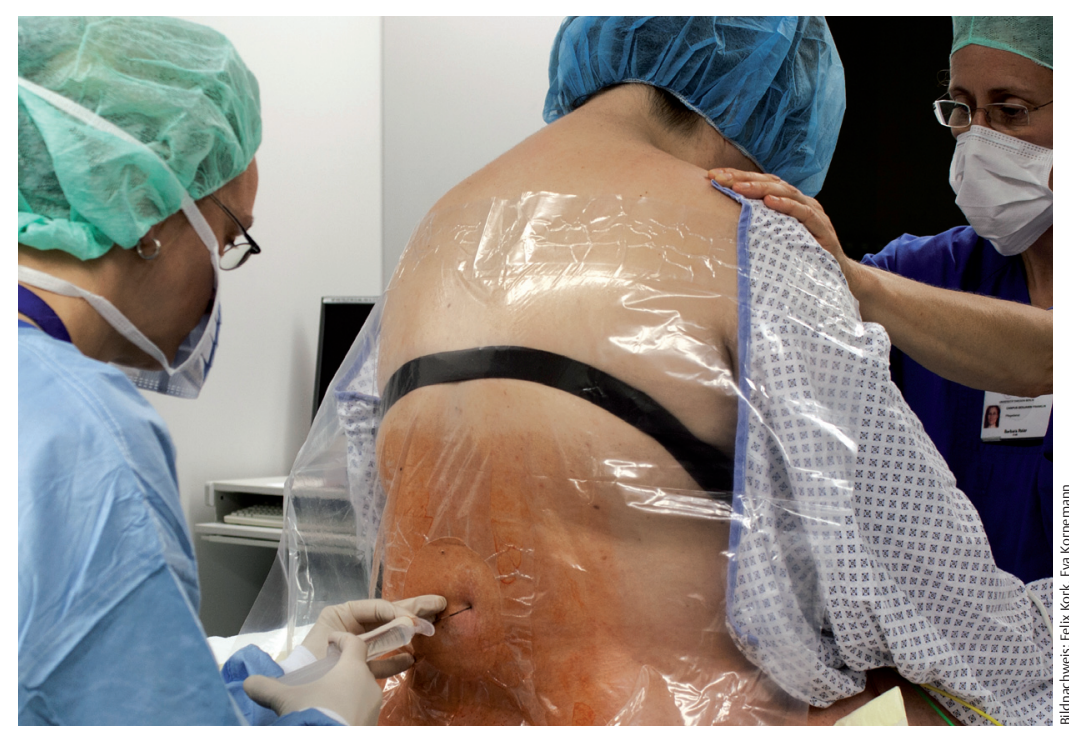




\section{Nichtopioid-Analgetika}

Vorsicht: Prostaglandinsynthese geht zurück Nichtsteroidale Antiphlogistika (NSAID), Metamizol und Paracetamol passieren leicht die Plazenta und inhibieren in unterschiedlichem Ausmaß durch unspezifische Hemmung der Zyklooxygenase (COX) die fetale Prostaglandinsynthese. Für die pränatale Paracetamoleinnahme besteht eine erhöhte Inzidenz an kindlichem Asthma, sofern die Mutter eine bestimmte Variante des Antioxidans-Gens aufweist [17].

- Eine reduzierte Prostaglandinsynthese ab der 30. Schwangerschaftswoche kann zu einem vorzeitigen Verschluss des Ductus arteriosus Botalli mit pulmonaler Hypertonie, folgender Rechtsherzhypertrophie sowie reduzierter Urinproduktion und Oligohydramnion führen.

- NSAID sind daher im letzten Trimenon kontraindiziert $[11,18]$.

Eine verminderte Prostaglandinsynthese kann zudem durch den wehenhemmenden Effekt die Geburt verzögern sowie durch Störung der Thrombozytenaggregation die Blutungsneigung fördern [11].

Am besten: Paracetamol Während der Schwangerschaft gilt Paracetamol als das Analgetikum und Antipyretikum der 1. Wahl, Azetylsalizylsäure als Substanz der 2. Wahl. Bezüglich der Einnahme von selektiven COX-II-Inhibitoren in der Schwangerschaft liegen keine ausreichenden Daten vor. Metamizol kann zu Blutbildveränderungen sowie dem vorzeitigen Verschluss des Ductus arteriosus führen. Es ist daher Mittel der 3. Wahl während der Schwangerschaft und sollte bei mütterlichen Veränderungen des Blutbildes vermieden werden.

\section{Anästhetika und Adjuvanzien}

\section{Volatile Anästhetika}

$\nabla$

Unerwünschte Wirkungen Alle Inhalationsanästhetika haben lipophile Eigenschaften und ein geringes Molekulargewicht. Sie passieren daher rasch die Plazenta und können dosisabhängig zu fetaler Depression führen.

- Eine dosisabhängige uterusrelaxierende Wirkung der volatilen Anästhetika kann bei MACWerten (minimale alveoläre Konzentration) ab $1-1,5$ beobachtet werden.

- Neonatale Depressionen kommen bei MACWerten von 1,0 bei Sectiones caesareae dagegen kaum vor, sind bei höheren Werten aber möglich.

- Teratogene Effekte sind bei Verwendung volatiler Anästhetika in gebräuchlichen Dosierungen beim Menschen nicht bekannt.
Bis zu einem MAC-Wert von 1,0 kann man Inhalationsanästhetika als unbedenklich einstufen und unter Beachtung der Nebenwirkungen einsetzen während der gesamten Schwangerschaft sowie in der Geburtshilfe [19].

Lachgas vermeiden Lachgas $\left(\mathrm{N}_{2} \mathrm{O}\right)$ zeichnet sich durch niedriges Molekulargewicht und geringe Lipophilie aus. Die Plazentabarriere wird rasch überwunden. Fetale bzw. neonatale Depressionen sind beschrieben und durch Diffusionshypoxie, wie auch direkte Wirkungen auf das fetale Gehirn, möglich. Durch Oxidation des Cobalamins erfolgt die Beeinflussung des Vitamin- $\mathrm{B}_{12}$-Stoffwechsels und Hemmung der Methioninsynthetase.

- Dies kann sich negativ auf die DNA-Synthese und die Ausbildung der Mitosespindeln auswirken und sollte daher zum Zeitpunkt der Organogenese (bis 2. Trimenon) in der frühen Phase der Schwangerschaft nicht erfolgen [20].

- Im Rahmen geburtshilflicher Eingriffe sollte die Verwendung unter Berücksichtigung möglicher neonataler Diffusionshypoxie erst nach der Abnabelung erfolgen.

\section{Injektionsanästhetika}

$\checkmark$

Thiopental ist geeignet Bislang konnten für Barbiturate keine potenziell teratogenen oder mutagenen Eigenschaften festgestellt werden. Aus diesem Grunde gelten die Barbiturate als sichere Medikamente zur Narkoseeinleitung während der Schwangerschaft oder zur Geburtshilfe. Eine Passage über die Plazenta in das fetale Blut erfolgt rasch. Eine neonatale Atemdepression ist möglich und dosisabhängig.

- Zur Geburtshilfe kann die Verwendung von 4-5 mg/kg Thiopental zur Narkoseinduktion mit der nötigen Vorsicht empfohlen werden.

Bedingt einsetzbar: Propofol Propofol ist sehr lipophil und daher auch leicht plazentagängig. Der Hersteller rät aufgrund mangelnder Studienlage von der Verwendung während der Schwangerschaft ab. Dosisabhängig kann es negative Auswirkungen durch Atemdepression und reduzierten Apgar-Werten des Neonaten verursachen. Teratogene oder mutagene Effekte wurden bislang nicht beobachtet.

- Die Gabe von $2 \mathrm{mg} / \mathrm{kg}$ zur Narkoseeinleitung oder die kontinuierliche Gabe bis $5 \mathrm{mg} / \mathrm{kg} / \mathrm{h}$ scheint geringe Auswirkungen auf die uteroplazentare Perfusion oder das postpartale Outcome des Neugeborenen zu haben.

- In den beschriebenen Dosierungen kann Propofol unter Beachtung der möglichen Nebenwirkungen in der Schwangerschaft und zur Geburtshilfe eingesetzt werden ( $\bullet$ Abb. 2). 
Etomidat von Vorteil bei Kreislaufinstabilität Das Hypnotikum Etomidat ist charakterisiert durch ein geringes Molekulargewicht und eine geringe Proteinbindung. Die Plazenta wird schnell überwunden. Etomidat zeichnet sich durch ausgeprägte hämodynamische Stabilität bei nur geringer Beeinflussung des Kreislaufs aus. Teratogene oder mutagene Nebenwirkungen sind nicht bekannt. Ebenso wurden bislang weder negative fetale noch neonatale Einflüsse beschrieben.

- Allerdings stellte man erniedrigte Serumkortisolspiegel des Neugeborenen durch eine reversible Hemmung der 11- $\beta$-Hydroxylase fest: Der enzymatisch katalysierte Syntheseschritt von 11- $\beta$-Desoxykortisol zu Kortisol wird gehemmt.

- Bereits eine einmalige Applikation von Etomidat schränkt die Kortisolsynthese signifikant ein $[21,22]$.

Diese Ergebnisse lassen sich sicherlich auch auf die neonatale Nebennierenrinde übertragen. Bei Narkoseeinleitung können exzitatorische Phänomene in Form von Myoklonien beobachtet werden, die ohne epileptoforme Korrelate im EEG ablaufen. Sie entstehen vermutlich durch Aktivierung von Neuronen im Hirnstamm oder tiefer gelegenen Strukturen (spinale Ebene).

- Wegen der Neutralität auf die Hämodynamik bietet sich die Verwendung besonders bei kreislaufinstabilen Patientinnen an - auch in der Schwangerschaft und Geburtshilfe.

- Über negative Auswirkung der Nebennierenrindensuppression des Neugeborenen ist bislang nichts bekannt.

\section{Lokalanästhetika}

Gefahr der Kumulation Lokalanästhetika weisen unterschiedliche Lipophilie, Plasmaeiweißbindung und je nach pKa-Wert unterschiedliche Ionisierungen auf. Je nach Konzentration, Menge und Perfusion der Gewebe erfolgt die Absorption in das mütterliche Blut. Von dort aus ist eine leichte Plazentapassage der basischen Substanzen möglich. Der fetale Mangel an $\alpha-1$ Glykoprotein erhöht den freien, ungebundenen Anteil des Lokalanästhetikums.

- Ein fetaler $\mathrm{pH}$ im azidotischen Bereich (bei Hypoxie, Asphyxie oder eingeschränkter uteroplazentarer Perfusion) bedingt einen höheren Ionisierungsgrad und führt aufgrund reduzierter Rückverteilung zur Kumulation, dem sog. „ion trapping“.

Eine Kumulation von Lokalanästhetika kann kardiotoxische und zentralnervöse systemische Nebenwirkungen verursachen.

Kein Prilocain! Mepivacain, Lidocain und Prilocain passieren leichter die Plazenta als Ropivacain und Bupivacain. Die üblichen Dosierungen bei rückenmarksnahen Verfahren führen zu keinen wesentlichen toxischen Effekten für den Fetus oder das Neugeborene - mit einer Ausnahme:

- Bei Prilocain ist besondere Vorsicht geboten! Es führt zur Methämoglobinämie, die sich beim Neugeborenen aufgrund mangelnder Aktivität der Zytochrom-b ${ }_{5}$-Reduktase schwerwiegender ausprägen kann [16].

- Prilocain sollte daher in Schwangerschaft und Geburtshilfe nicht mehr eingesetzt werden!

Ropivacain und Bupivacain In klinisch üblichen Dosierungen kann der Einsatz von Ropivacain und Bupivacain unter Beachtung der toxischen Konzentrationen ( $\bullet$ Tab. 3 ) für die spinale und epidurale Anwendung empfohlen werden.

- Ropivacain bietet sich im Vergleich zu Bupivacain aufgrund der größeren therapeutischen Breite und geringeren Kardiotoxizität an [4].

- Eine Kombination mit Opiaten kann ggf. die effektive Dosis und damit auch toxische Komplikationen reduzieren.

\section{Benzodiazepine \\ $\boldsymbol{\nabla}$}

Risiken Benzodiazepine sind unterschiedlich lipophil und gelangen leicht über die Plazenta in den fetalen Kreislauf. Da die fetale Metabolisierung nur eingeschränkt funktioniert, können die Substanzen kumulieren. Unter Umständen sind pharmakologisch aktive Metabolite noch nach Tagen und Wochen im fetalen Gewebe nachweisbar. - Benzodiazepine können zum sog. „Floppy-Infant-Syndrom" führen. Es ist gekennzeichnet durch Atemdepression, Hyporeflexie, schlaffem Muskeltonus, Thermoregulationsstörungen und vermindertem Apgar-Wert beim Neugeborenen.

Allenfalls in der späten Schwangerschaft Der Einsatz von Benzodiazepinen im 1. Trimenon kann das Risiko von Spaltbildungen im Gesichtsbereich um das 10 -Fache von $0,06 \%$ auf etwa $0,7 \%$ erhöhen [23].

- Eine Einnahme in der Frühschwangerschaft sollte vermieden werden.

\section{Toxische Dosierungen von Lokalanästhetika}

Bupivacain: 2-3 mg/kg KG

- Gegen die sachgemäße Anwendung niedrig konzentrierter BupivacainLösungen zur PDA $(0,125-0,25 \%)$ oder $0,5 \%$ iger Lösungen zur Spinalanästhesie gibt es kaum Einwände.

- Cave: Höher konzentrierte Lösungen (0,75\%) haben eine geringe therapeutische Breite bei gleichzeitiger Kardio- und Neurotoxizität! Es sind schwerwiegende Herzrhythmusstörungen im Rahmen der Geburtshilfe beschrieben (häufig Überdosierungen und teils intravasale Applikation).

Ropivacain: 3-4 mg/kg KG

- Nur zur epiduralen Anwendung!

- Für die intrathekale Applikation besteht keine Zulassung. 
Zudem besteht bei Einnahme in der Schwangerschaft Suchtpotenzial für Mutter und Kind.

- In der späteren Schwangerschaft kann unter strenger Indikationsstellung die Gabe von kurzwirksamen und gering lipophilen Benzodiazepinen (z.B. Midazolam) erfolgen.

- Von einer Prämedikation vor einer Sectio caesarea wird abgeraten.

\section{Ketamin}

$\nabla$

Gut - aber erst im 3. Trimenon Ketamin ist als racemisches Gemisch (S-, R-) und als S-Enantiomer lipophil und mit geringem Molekulargewicht leicht plazentagängig. Maternal können neben Blutdruckanstiegen, Hypersalivation, motorischer Unruhe und psychotropen Effekten auch uterine Kontraktionen ausgelöst werden.

- Eine Kombination mit einem Benzodiazepin ist sinnvoll, in der Schwangerschaft jedoch nicht optimal (s.oben).

- Bei der Verwendung von Ketamin zur Geburtshilfe sind die geringsten Raten an Awareness sowie die Aufrechterhaltung stabiler Kreislaufverhältnisse beschrieben.

- Bei Asthma, chronischen Lungenerkrankungen, bei instabiler Hämodynamik oder septischem Schock bietet sich Ketamin als Einleitungsnarkotikum (1-2 mg/kg KG) an.

Praxistipp Applizieren Sie dem Neugeborenen nach der Entwicklung 0,05-0,1 mg/kg Midazolam, um psychotropen Effekten vorzubeugen.

Wann ist Ketamin kontraindiziert? Ein Einsatz von Ketamin in der Frühschwangerschaft (1. und 2. Trimenon) wird wegen der Steigerung des basalen Uterotonus nicht empfohlen. Auch stellen arterielle Hypertonien (auch schwangerschaftsinduziert) und Präeklampsie absolute Kontraindikationen dar [19].

\section{Muskelrelaxanzien}

\section{$\nabla$}

Kaum plazentagängig Als stark ionisierte und hydrophile Substanzen passieren Muskelrelaxanzien nur in geringem Ausmaß die Plazenta. Etwa $10 \%$ der mütterlichen Konzentration lassen sich im fetalen Kreislauf feststellen.

Relativ sicher Von einzelnen Atemdepressionen des Neugeborenen bei der Verwendung von Succinylcholin ist berichtet worden. Auch eine Erhöhung des Uterotonus mit eventueller Stimulation der Wehentätigkeit ist nach der Verwendung von Succinylcholin möglich [11].

- Für nicht depolarisierende Muskelrelaxanzien sind bislang in klinisch üblichen Dosierungen keine fetalen oder neonatalen Depressionen beschrieben.

- Unter Beachtung möglicher Gegenanzeigen kann die Gabe von Muskelrelaxanzien (auch Succinylcholin) in klinisch üblicher Dosierung erfolgen.

- Teratogene oder mutagene Auswirkungen sind nicht bekannt.

Reversierung Um die Wirkung nicht depolarisierender Muskelrelaxanzien aufzuheben, steht für Rocuronium und Vecuronium das modifizierte $\gamma$-Cyclodextrin Sugammadex zur Verfügung.

- Über die Verwendung von Sugammadex in der Schwangerschaft liegen keine Daten vor.

- Tierexperimentell gibt es keine Hinweise auf embryotoxische Wirkungen.

\section{Vasoaktive Substanzen}

\section{Antihypertonika}

Geeignete Substanzen In der Schwangerschaft als empfehlenswert erachtet werden die Vasodilatatoren und $\alpha$-Rezeptor-Antagonisten Urapidil, Dihydralazin und Diazoxin. Für zentral wirksame antiadrenerge Substanzen wie Clonidin, Moxonidin und $\alpha$-Methyldopa gibt es widersprüchliche Empfehlungen [24, 25].

- In der Therapie der schwangerschaftsinduzierten Hypertonie (SIH) bevorzugt man derzeit Dihydralazin und $\alpha$-Methyldopa.

- Mit dem Vorteil der peroralen Einnahme kann man auch $\beta$-Blocker und Kalziumantagonisten während der Schwangerschaft einsetzen.

Darauf sollten Sie achten Auswirkungen auf den Feten ergeben sich durch mögliche Bradykardien, Wachstumsretardierungen und neonatale Hypoglykämien [2, 16].

- Kontraindiziert sind ACE-Hemmer und Angiotensin-II-Rezeptor-Antagonisten aufgrund ihres teratogenen Potenzials (Anurie, Schädelhypoplasie, Oligohydramnion)

- sowie Na-Nitroprussid aufgrund möglicher fetaler Zyanidbelastung.

Vor Einnahme dieser kontraindizierten Antihypertensiva sollte eine Schwangerschaft ausgeschlossen werden. Bei einer geplanten Konzeption muss eine entsprechende Alternativtherapie erfolgen.

Diuretika: nur im Spezialfall Die Verabreichung von Diuretika ist wegen des Einflusses auf fetales Wachstum und Elektrolythaushalt relativ kontraindiziert und nur speziellen Indikationen vorbehalten.

- Sollte es dennoch notwendig werden, ein Diuretikum einzusetzen, dann sollte Hydrochlorothiazid Anwendung finden. 


\section{Vasokonstriktoren}

Auf direkte $\alpha$-Sympathomimetika verzichten Beachten Sie bei der Therapie maternaler Hypotensionen, dass Vasokonstriktoren auf Basis $\alpha$-adrenerger Stimulation die uteroplazentare Perfusion negativ beeinträchtigen.

- Meiden Sie daher Sympathomimetika mit direkter $\alpha$-adrenerger Wirkung oder setzen Sie diese nur mit äußerster Vorsicht ein.

- Die Verwendung von Akrinor ${ }^{\circledR}$ - einem Kombinationspräparat aus Theodrenalin und Cafedrin mit vornehmlich $\beta$-adrenergen Effekten und geringerer Beeinträchtigung der uteroplazentaren Perfusion - kann empfohlen werden [2].

- Auch für den Einsatz von Ephedrin in niedriger Dosierung zur Steigerung des arteriellen Druckes gibt es keine Einwände.

Cave Von dem Gebrauch von Etilefrin wird abgeraten. Es sind teratogene Effekte beschrieben [2].

\section{Antikoagulanzien}

Am besten Heparin Das Mittel der Wahl zur Antikoagulation während der Schwangerschaft ist unfraktioniertes Heparin. Aufgrund der Molekülgröße kommt es nicht zur Passage der Plazenta. Auch für niedermolekulare Heparine besteht die plazentare Barriere. Eine Anwendung scheint unbedenklich, sowie das Risiko für eine heparininduzierte Thrombozytopenie (HIT) und Osteoporose reduziert [26].

Bloß keine Cumarine! Cumarinderivate sind aufgrund ihrer teratogenen Wirkung absolut kontraindiziert. Bei Einnahme während der Schwangerschaft kann eine typische Embryopathie resultieren (in 15-30\%), die sich kennzeichnet durch

- Hypoplasien der Knochen,

- vorzeitige Epiphysenkalzifizierung und

- Entwicklungsretardierung.

- Zudem kann es zu intrazerebralen Blutungen unter der Geburt kommen [1].

Dr. med. Dirk Nauheimer D. E. S. A. ist Facharzt für Anästhesie mit Zusatzbezeichnung Notfallmedizin. E arbeitet am Krankenhaus der Barmherzigen Brüder in Trier. E-Mail: dirk.nauheimer@gmx.de

Interessenkonflikt Der Autor erklärt, dass keine Interessenkonflikte vorliegen.

Beitrag online zu finden unter http://dx.doi. org/10.1055/s-0032-1330932

\section{Kernaussagen}

- Für nahezu alle Medikamentenapplikationen während der Schwangerschaft kann von einer Passage der Plazenta und folglich auch einer Wirkung auf den Feten ausgegangen werden.

- Anästhesieverfahren während der Schwangerschaft oder zur Geburtshilfe können mit erhöhtem Risiko und nachteiligen Auswirkungen für Mutter und Kind einhergehen.

$\triangleright$ Sofern möglich, sollten Eingriffe außerhalb der sensiblen Phase der Organogenese im 1. Trimenon erfolgen.

$\triangleright$ Ab dem 2. und 3. Trimenon erhöht sich das Risiko einer Frühgeburt.

- Um möglichst geringe Auswirkungen auf die uteroplazentare Perfusion auszuüben, sollte die Auswahl der einzusetzenden Medikamente vorsichtig erfolgen.

$\triangleright$ Für viele Indikationen lassen sich geeignete Präparate finden, die ein möglichst geringes Risiko für den Fetus oder den Neonaten bergen.

- Bei Unklarheit über die Verwendung spezieller Präparate hilft auch die Suche über das Internetportal o www.embryotox.de weiter.

Zudem ist die Erfahrung von Geburtshelfern und behandelnden Disziplinen, ggf auch Pädiatern, oft von unschätzbarem Wert. Streben Sie daher eine interdisziplinäre Zusammenarbeit unbedingt an.

\section{Literatur}

1 Schaefer C, Weber-Schöndorfer C. Medikamentöse Therapie in der Schwangerschaft. Dtsch Arztebl 2005; 102: 2480-2488

2 Schott C, Schmidt H. General anesthesia in pregnancy. Anaesthesist 1998; 47: 525-536

3 Stamer UM, Stuber F, Wiese R et al. Contraindications to regional anaesthesia in obstetrics: a survey of German practice. Int I Obstet Anesth 2007; 16: 328-335

4 Bachmann-Mennenga B, Veit G. Anästhesietechniken zur Sectio caesarea - Katheter-Periduralanästhesie. Anasthesiol Intensivmed Notfallmed Schmerzther 2001; 36: 53-57

5 Dahl V, Spreng UJ. Anaesthesia for urgent (grade 1) caesarean section. Curr Opin Anaesthesiol 2009; 22: 352-356

6 Stamer UM, Messerschmidt A, Wulf H. Anaesthesia for caesarean section - a German survey. Acta Anaesthesiol Scand 1998; 42: 678-684

7 Wulf H, Stamer U. Current practices in obstetrical analgesia in German university clinics. Results of a 1996 survey. Part 2. Anaesthesist 1998; 47: 757-764

8 Kort B, Katz VL, Watson WJ. The effect of nonobstetric operation during pregnancy. Surg Gynecol Obstet 1993; 177: 371-376

9 Cohen-Kerem R, Railton C, Oren D et al. Pregnancy outcome following non-obstetric surgical intervention. Am J Surg 2005; 190: 467-473

10 Engels K. Physiological changes during pregnancy relevant to anesthesia. Anasthesiol Intensivmed Notfallmed Schmerzther 2001; 36: 39-42

11 Gogarten W, van Aken H. Anästhesie während der Schwangerschaft. Anesthesiol Intensivmed 2001; 42: 963-972

12 Lang C, Behnke H, Wulf H, Geldner G. Placental passage of anesthetics and adjuvants. Anaesthesist 2002; 51: 409-417

13 von Neindorff M. Fetomaternal pharmacology: anesthesiological approach in surgical interventions during pregnancy. Anaesthesist 59: 479-490

14 Nahum GG, Uhl K, Kennedy DL. Antibiotic use in pregnancy and lactation: what is and is not known about teratogenic and toxic risks. Obstet Gynecol 2006; 107: 1120-1138

15 Schlack W, Küppers V, Lorenz C. Anästhesie für operative Eingriffe während der Schwangerschaft. Gynäkologe 1997; 30: 835-840

\section{Literatur online}

Das vollständige Literaturverzeichnis zu diesem Beitrag finden Sie im Internet:

Abonnenten und Nichtabonnenten können unter „www.thieme-connect.de/ ejournals“ die Seite der Lege artis aufrufen und beim jeweiligen Artikel auf „Zusatzmaterial“" klicken - hier ist die Literatur für alle frei zugänglich.

Abonnenten können alternativ über ihren persönlichen Zugang an das Literaturverzeichnis gelangen. Wie das funktioniert, lesen Sie unter: http://www.thiemeconnect.de/ejournals/help\#SoRegistrieren 